\title{
Generation of atomic entangled states in a bi-mode cavity via adiabatic passage
}

\author{
Li-Bo Chen, Peng Shi, Yong-Jian Gu* Lin Xie, and Li-Zhen Ma \\ Department of Physics, Ocean University of China, Qingdao 266100, People's Republic of China
}

\begin{abstract}
We propose schemes to prepare atomic entangled states in a bi-mode cavity via stimulated Raman adiabatic passage (STIRAP) and fractional stimulated Raman adiabatic passage (f-STIRAP) techniques. According to the simulation results, our schemes keep the cavity modes almost unexcited and the atomic excited states are nearly unpopulated during the whole process. The simulation also shows that the error probability is very small.
\end{abstract}

\section{INTRODUCTION}

Entanglement, especially high dimensional entanglement, has been recognized as the crucial ingredient in many quantum information processes, such as quantum communication [1, 2, 3, 4, 4, 5], quantum computation [6, 7, 8]. Thus more and more attention has been paid to generating qubit or qudit entangled states in cavity QED [9, 10, 11, 12, 13, 14, 15, 16, 17], ion trap [18, 19, 20], superconducting qubit [21, 22, 23, 24], linear optical system [25, 26], semiconductor quantum dot [27, 28], and so on.

The technique of stimulated Raman adiabatic passage (STIRAP), which was shown theoretically [29] and implemented experimentally in population transfer process in molecules and atoms [30, 31], has been broadly used in quantum information processing (QIP) [32, 33, 34, 35, 36, 37, 38, 39, 40, 41, 42]. In this technique are involved two delayed, but partly overlapping pulses - pump and Stokes - with the Stokes pulse applied first. L. Wang et al [43] experimentally and theoretically demonstrate that the atomic coherence can be completely transferred or arbitrarily distributed among the different levels in a four-level atomic (tripod) scheme by STIRAP. An extension of STIRAP, called fractional stimulated Raman adiabatic passage (f-STIRAP) [44, 45, 46, 47], allows the creation of a coherent superposition of two ground states in $\Lambda$-type system. Different from STIRAP, the f-STIRAP requires that the two pulses vanish at a constant finite ratio of amplitudes. Wang et al [48] have demonstrated storage and selective release of a light pulse in a Pr:YSO crystal, which is based on atomic spin coherence created by the f-STIRAP.

In this Brief Report, we present schemes for generating entangled states of two qubits and two qutrits via STIRAP or f-STIRAP techniques. Two ${ }^{87} R b$ atoms are trapped in an optical cavity and drived by two different lasers. By choosing appropriate parameters we can create entangled atomic states. According to the simulation results, our schemes keep the cavity modes almost unexcited and the atomic excited states are nearly unpopulated during the whole process. The simulation also shows that the error probability is very small.

\section{THE FUNDAMENTAL MODEL}

We consider the situation described in Figure 1, where two atoms are trapped in a bi-mode optical cavity. The relevant atomic levels and transitions are also depicted in this figure; such level structures can be achieved in ${ }^{87} R b$ 49, [50]. The states $\left|g_{L}\right\rangle,\left|g_{0}\right\rangle,\left|g_{R}\right\rangle$ and $\left|g_{a}\right\rangle$ correspond to ${ }^{87} R b$ atom hyperfine levels $\left|F=1, m_{F}=-1\right\rangle,\left|F=1, m_{F}=0\right\rangle$, $\left|F=1, m_{F}=1\right\rangle$ of $5 S_{1 / 2}$ and $\left|F=2, m_{F}=0\right\rangle$ of $5 S_{1 / 2}$, while $\left|e_{L}\right\rangle,\left|e_{0}\right\rangle$ and $\left|e_{R}\right\rangle$ correspond to $\left|F=1, m_{F}=-1\right\rangle$, $\left|F=1, m_{F}=0\right\rangle$ and $\left|F=1, m_{F}=1\right\rangle$ of $5 P_{3 / 2}$. Initially, the atoms $A$ and $B$ are prepared in the state $\left|g_{a}\right\rangle_{A}$ and $\left|g_{0}\right\rangle_{B}$ respectively, and the cavity mode is in the vacuum state. The atom $A$ 's transition $\left|g_{a}\right\rangle_{A} \leftrightarrow\left|e_{0}\right\rangle_{A}$ is driven resonantly by a $\pi$-polarized classical field with Rabi frequency $\Omega_{A} ;\left|e_{0}\right\rangle_{A} \leftrightarrow\left|g_{R}\right\rangle_{A}\left(\left|e_{0}\right\rangle_{A} \leftrightarrow\left|g_{L}\right\rangle_{A}\right)$ is resonantly coupled to the cavity mode $a_{R}\left(a_{L}\right)$ with coupling constant $g_{A}$. The atom $B$ 's transition $\left|g_{L}\right\rangle_{B} \leftrightarrow\left|e_{L}\right\rangle_{B}\left(\left|g_{R}\right\rangle_{B} \leftrightarrow\left|e_{R}\right\rangle_{B}\right)$ is driven resonantly by a $\pi$-polarized classical field with Rabi frequency $\Omega_{B} ;\left|e_{L}\right\rangle_{B} \leftrightarrow\left|g_{0}\right\rangle_{B}\left(\left|e_{R}\right\rangle_{B} \leftrightarrow\left|g_{0}\right\rangle_{B}\right)$ is resonantly coupled to the cavity mode $a_{R}\left(a_{L}\right)$ with coupling constant $g_{B}$. In the rotating wave approximation, the interaction Hamiltonian can be written as (setting $\hbar=1$ )

$$
\begin{aligned}
H_{0} & =g_{A}(t) a_{R}\left|e_{0}\right\rangle_{A}\left\langle g_{R}\left|+g_{A}(t) a_{L}\right| e_{0}\right\rangle_{A}\left\langle g_{L}\left|+\Omega_{A}(t)\right| e_{0}\right\rangle_{A}\left\langle g_{a}\right| \\
& +g_{B}(t) a_{R}\left|e_{L}\right\rangle_{B}\left\langle g_{0}\left|+g_{B}(t) a_{L}\right| e_{R}\right\rangle_{B}\left\langle g_{0}\left|+\Omega_{B}(t)\right| e_{L}\right\rangle_{B}\left\langle g_{L}\left|+\Omega_{B}(t)\right| e_{R}\right\rangle_{B}\left\langle g_{R}\right|+H . c . .
\end{aligned}
$$

*Electronic address: yjgu@ouc.edu.cn 


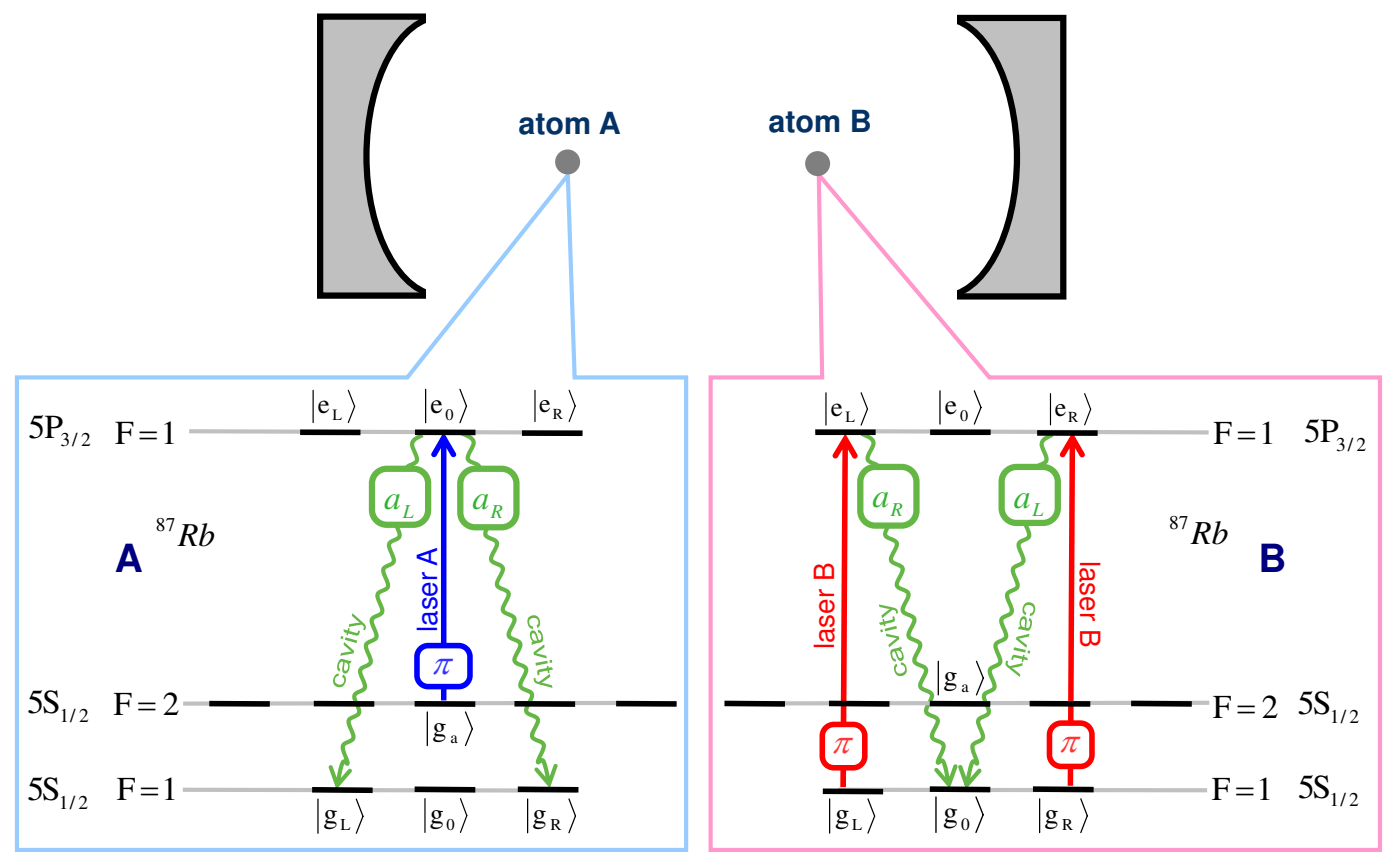

FIG. 1: Two ${ }^{87} R b$ atoms are trapped in a bi-mode cavity. The states $\left|g_{L}\right\rangle\left(\left|e_{L}\right\rangle\right),\left|g_{0}\right\rangle\left(\left|e_{0}\right\rangle\right)$, and $\left|g_{R}\right\rangle\left(\left|e_{R}\right\rangle\right)$ correspond to the ${ }^{87} R b$ atom hyperfine levels $F=1$ of $5 S_{1 / 2}\left(5 P_{3 / 2}\right)$, while $\left|g_{a}\right\rangle$ corresponds to $F=2, m_{F}=0$ of $5 S_{1 / 2}$. The atom $A$ 's transition $\left|g_{a}\right\rangle_{A} \leftrightarrow\left|e_{0}\right\rangle_{A}$ is driven resonantly by a $\pi$-polarized classical field with Rabi frequency $\Omega_{A} ;\left|e_{0}\right\rangle_{A} \leftrightarrow\left|g_{R}\right\rangle_{A}\left(\left|e_{0}\right\rangle_{A} \leftrightarrow\left|g_{L}\right\rangle_{A}\right)$ is resonantly coupled to the cavity mode $a_{R}\left(a_{L}\right)$ with coupling constant $g_{A}$. The atom $B$ 's transition $\left|g_{L}\right\rangle_{B} \leftrightarrow\left|e_{L}\right\rangle_{B}$ $\left(\left|g_{R}\right\rangle_{B} \leftrightarrow\left|e_{R}\right\rangle_{B}\right)$ is driven resonantly by a $\pi$-polarized classical field with Rabi frequency $\Omega_{B} ;\left|e_{L}\right\rangle_{B} \leftrightarrow\left|g_{0}\right\rangle_{B}\left(\left|e_{R}\right\rangle_{B} \leftrightarrow\left|g_{0}\right\rangle_{B}\right)$ is resonantly coupled to the cavity mode $a_{R}\left(a_{L}\right)$ with coupling constant $g_{B}$.

In the following we write the state of the system as $\left|A, B, n_{R}, n_{L}\right\rangle$, where $A$ and $B$ denote the states of the atoms, and $n_{L . R}$ the number of $L$ or $R$ polarized photons of the cavity. The subspace $S$ spanned by states $\left\{\left|g_{a}, g_{0}, 0,0\right\rangle\right.$, $\left.\left|e_{0}, g_{0}, 0,0\right\rangle,\left|g_{L}, g_{0}, 0,1\right\rangle,\left|g_{L}, e_{R}, 0,0\right\rangle,\left|g_{L}, g_{R}, 0,0\right\rangle,\left|g_{R}, g_{0}, 1,0\right\rangle,\left|g_{R}, e_{L}, 0,0\right\rangle,\left|g_{R}, g_{L}, 0,0\right\rangle\right\}$ is an 8-dimensionnal invariant subspace of the Hamiltonian (1). It can be verified that in the subspace $S$, the Hamiltonian has the following dark state:

$$
\begin{aligned}
|D(t)\rangle & =C\left[2 g_{A}(t) \Omega_{B}(t)\left|g_{a}, g_{0}, 0,0\right\rangle-\Omega_{A}(t) \Omega_{B}(t)\left(\left|g_{L}, g_{0}, 0,1\right\rangle+\left|g_{R}, g_{0}, 1,0\right\rangle\right)\right. \\
& \left.+g_{B}(t) \Omega_{A}(t)\left(\left|g_{L}, g_{R}, 0,0\right\rangle+\left|g_{R}, g_{L}, 0,0\right\rangle\right)\right]
\end{aligned}
$$

where we assume $g_{i}, \Omega_{i}$ are real, and $C^{-2}=4 g_{A}^{2} \Omega_{B}^{2}+2 \Omega_{A}^{2} \Omega_{B}^{2}+2 g_{B}^{2} \Omega_{A}^{2}$. Under the condition

$$
g_{A}(t), g_{B}(t) \gg \Omega_{A}(t), \Omega_{B}(t),
$$

we have

$$
|D(t)\rangle \sim 2 g_{A}(t) \Omega_{B}(t)\left|g_{a}, g_{0}, 0,0\right\rangle+g_{B}(t) \Omega_{A}(t)\left|g_{L}, g_{R}, 0,0\right\rangle+g_{B}(t) \Omega_{A}(t)\left|g_{R}, g_{L}, 0,0\right\rangle
$$

\section{GENERATION OF A TWO-QUBIT ENTANGLED STATE VIA STIRAP}

Suppose the initial state of the system is $\left|g_{a}, g_{0}, 0,0\right\rangle$, if we design pulse shapes and sequence such that

$$
\begin{aligned}
& \lim _{t \rightarrow-\infty} \frac{g_{B}(t) \Omega_{A}(t)}{g_{A}(t) \Omega_{B}(t)}=0, \\
& \lim _{t \rightarrow+\infty} \frac{g_{A}(t) \Omega_{B}(t)}{g_{B}(t) \Omega_{A}(t)}=0,
\end{aligned}
$$


it follows from Eq. (4) that we can adiabatically transfer the initial state $\left|g_{a}, g_{0}, 0,0\right\rangle$ to a superposition of $\left|g_{L}, g_{R}, 0,0\right\rangle$ and $\left|g_{R}, g_{L}, 0,0\right\rangle$, i.e., $1 / \sqrt{2}\left(\left|g_{L}, g_{R}, 0,0\right\rangle+\left|g_{R}, g_{L}, 0,0\right\rangle\right)=1 / \sqrt{2}\left(\left|g_{L}, g_{R}\right\rangle+\left|g_{R}, g_{L}\right\rangle\right)|00\rangle_{c}$, which is a product state of the two-atom entangled state and the cavity mode vacuum state.

The pulse shapes and sequence can be designed by an appropriate choice of the parameters. The coupling rates are chosen such that $g_{A}(t)=g_{B}(t)=g$, laser Rabi frequencies are chosen as $\Omega_{A}(t)=\Omega_{0} \exp \left[-1 / 200\left(t-t_{0}\right)^{2} / \tau^{2}\right]$ and $\Omega_{B}(t)=\Omega_{0} \exp \left[-1 / 200 t^{2} / \tau^{2}\right]$, with $t_{0}=2 \tau$ being the delay between pulses [32]. With this choice, conditions (5) and (6) are satisfied.

To evaluate the performance of our scheme, we now consider the dissipative processes due to spontaneous decay of the atoms from the excited states and the decay of cavity. The evolution of the system is governed by the nonHermitian Hamiltonian

$$
H_{n h}=H_{0}-i \kappa\left(a_{L}^{+} a_{L}+a_{R}^{+} a_{R}\right)-\gamma \sum_{j=A, B}\left(\left|e_{R}\right\rangle_{j}\left\langle e_{R}|+| e_{L}\right\rangle_{j}\left\langle e_{L}|+| e_{0}\right\rangle_{j}\left\langle e_{0}\right|\right),
$$

where $\kappa$ is the cavity decay rate and $\gamma$ is the atomic spontaneous emission rate. Here we assume that three excited states $\left|e_{R}\right\rangle,\left|e_{L}\right\rangle$, and $\left|e_{0}\right\rangle$ have the same spontaneous emission rate $\gamma$, and the two cavity modes possess the same loss rate $\kappa$. Figure 2 shows the simulation results of the two-qubit entanglement generation process, where we choose $g=5 \Omega_{0}, \tau=\Omega_{0}^{-1}$, the cavity decay rate and the atomic spontaneous emission rate $\kappa=\gamma=0.005 g$ [51, 52]. The Rabi frequencies of $\Omega_{A}(t), \Omega_{B}(t)$ are shown in Fig. 2(a). Figure 2(b) shows the time evolution of populations, in which $P_{1}, P_{5}$, and $P_{8}$ denote the populations of the states $\left|g_{a}, g_{0}, 0,0\right\rangle,\left|g_{L}, g_{R}, 0,0\right\rangle$, and $\left|g_{R}, g_{L}, 0,0\right\rangle$, and the populations of the states $\left\{\left|e_{0}, g_{0}, 0,0\right\rangle,\left|g_{L}, g_{0}, 0,1\right\rangle,\left|g_{L}, e_{R}, 0,0\right\rangle,\left|g_{R}, g_{0}, 1,0\right\rangle,\left|g_{R}, e_{L}, 0,0\right\rangle\right\}$ are almost zero during the whole dynamics. Finally $P_{5}$ and $P_{8}$ arrive at 0.5 and $P_{1}$ approaches 0 , which means the successful generation of the two qubit entangled state. Figure 2(c) shows the error probability defined by [53]:

$$
P_{e}=1-\left|\left\langle D(t) \mid \varphi_{s}(t)\right\rangle\right|^{2}
$$

here $\left|\varphi_{s}(t)\right\rangle$ is the state obtained by the simulation and $|D(t)\rangle$ is the dark state defined by Eq. (2). In Figure 2(d), the probability $P_{p}$ with which one photon appears in the cavity is shown. Figure $2(\mathrm{e})$ shows the population $P_{e a}$ of the atoms in excited state $\left|e_{i}\right\rangle(i=L, 0, R)$. From Figures 2(c)-2(e) we conclude that we can prepare the two qubit entangled state with high success probability.

\section{GENERATION OF A TWO-QUTRIT ENTANGLED STATE VIA F-STIRAP}

Suppose the initial state of the system is $\left|g_{a}, g_{0}, 0,0\right\rangle$, if we design pulse shapes and sequence such that

$$
\begin{aligned}
& \lim _{t \rightarrow-\infty} \frac{g_{B}(t) \Omega_{A}(t)}{g_{A}(t) \Omega_{B}(t)}=0, \\
& \lim _{t \rightarrow+\infty} \frac{g_{A}(t) \Omega_{B}(t)}{g_{A}(t) \Omega_{B}(t)}=\frac{1}{2},
\end{aligned}
$$

the system will end up in the state $1 / \sqrt{3}\left(\left|g_{a}, g_{0}, 0,0\right\rangle+\left|g_{L}, g_{R}, 0,0\right\rangle+\left|g_{R}, g_{L}, 0,0\right\rangle\right)=1 / \sqrt{3}\left(\left|g_{a}, g_{0}\right\rangle+\left|g_{L}, g_{R}\right\rangle+\right.$ $\left.\left|g_{R}, g_{L}\right\rangle\right)|00\rangle_{c}$, which is a product state of the three-dimensional entangled state of the two atoms and the cavity mode vacuum state. We choose the pulses $g_{A}(t), \Omega_{A}(t), g_{B}(t)$, and $\Omega_{B}(t)$ the same as in Sec. III, i.e. $g_{A}(t)=g_{B}(t)=g$, $\Omega_{A}(t)=\Omega_{0} \exp \left[-1 / 200\left(t-t_{0}\right)^{2} / \tau^{2}\right]+\frac{\Omega_{0}}{2} \exp \left[-1 / 200 t^{2} / \tau^{2}\right]$ and $\Omega_{B}(t)=\Omega_{0} \exp \left[-1 / 200 t^{2} / \tau^{2}\right]$, with $t_{0}=2 \tau$.

Figure 3 shows the simulation results of the two-qutrit entanglement generation process, where we choose $g, \Omega_{0}, \tau, \kappa$, and $\gamma$ the same as in Figure 2. The Rabi frequencies of $\Omega_{A}(t), \Omega_{B}(t)$ are shown in Fig. 3(a). Figure 3(b) shows the time evolution of populations, in which $P_{1}, P_{5}$, and $P_{8}$ denote the populations of the states $\left|g_{a}, g_{0}, 0,0\right\rangle,\left|g_{L}, g_{R}, 0,0\right\rangle$, and $\left|g_{R}, g_{L}, 0,0\right\rangle$, and the populations of the states $\left\{\left|e_{0}, g_{0}, 0,0\right\rangle,\left|g_{L}, g_{0}, 0,1\right\rangle,\left|g_{L}, e_{R}, 0,0\right\rangle,\left|g_{R}, g_{0}, 1,0\right\rangle,\left|g_{R}, e_{L}, 0,0\right\rangle\right\}$ are almost zero during the whole dynamics. Finally $P_{1}, P_{5}$, and $P_{8}$ arrive at $1 / 3$, which means the successful generation of the two qutrit entangled state. Figure 3(c) shows the error probability during the process. In Figure 3(d), the probability $P_{p}$ with which one photon appears in the cavity is shown. Figure $3(\mathrm{e})$ shows the population $P_{e a}$ of the atoms in excited state $\left|e_{i}\right\rangle(i=L, 0, R)$. From Figures 3(c)-3(e) we conclude that we can prepare the two qutrit entangled state with high success probability. 


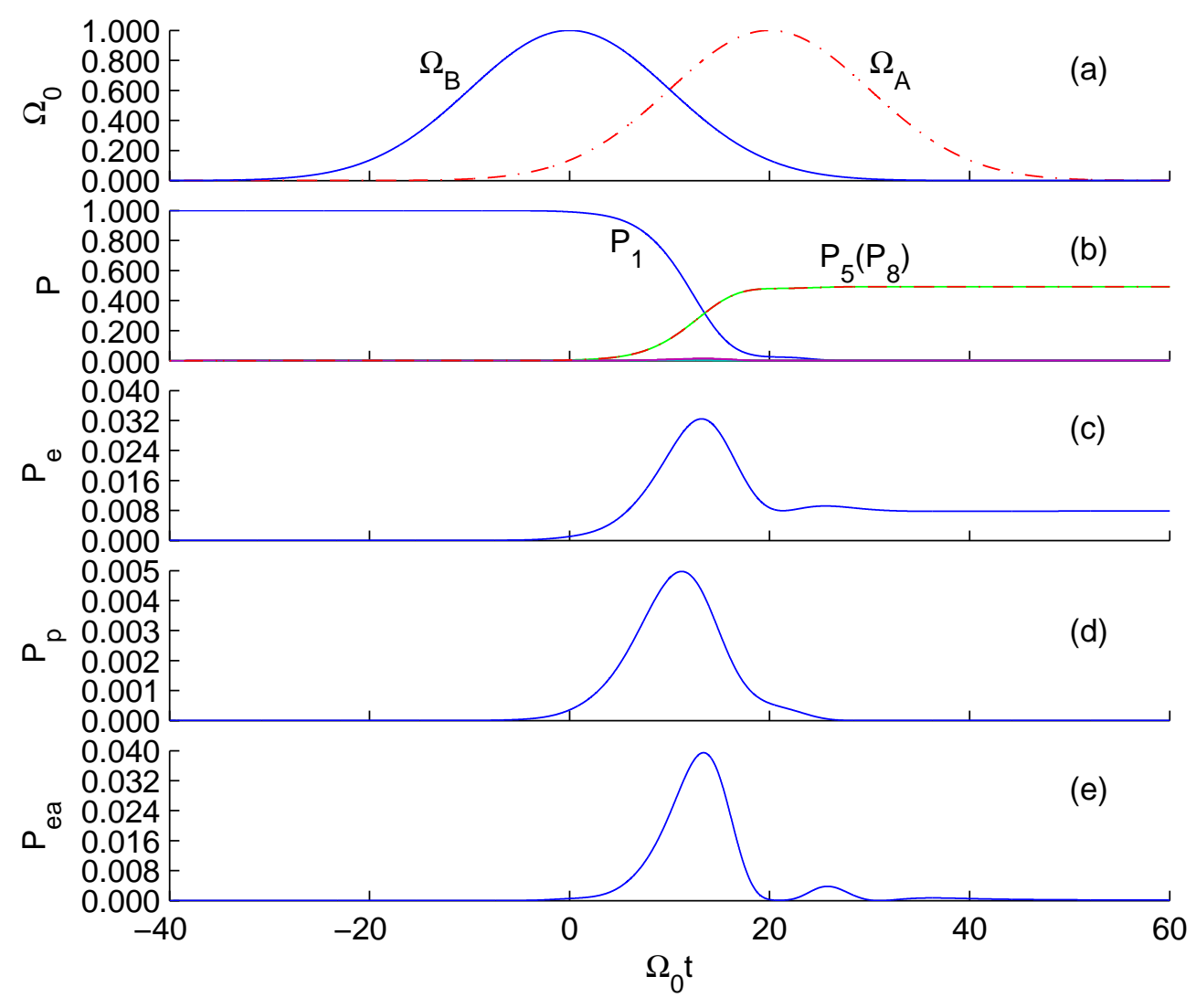

FIG. 2: The simulation results of the two-qubit entanglement generation process, where we choose $g=5 \Omega_{0}, \tau=\Omega_{0}^{-1}$, the cavity decay rate and the atomic spontaneous emission rate $\kappa=\gamma=0.005 \mathrm{~g}$. Figure 2(a): the Rabi frequency of $\Omega_{A}(t), \Omega_{B}(t)$. Figure 2(b): the time evolution of populations, in which $P_{1}, P_{5}$, and $P_{8}$ denote the populations of the states $\left|g_{a}, g_{0}, 0,0\right\rangle,\left|g_{L}, g_{R}, 0,0\right\rangle$, and $\left|g_{R}, g_{L}, 0,0\right\rangle$, and the populations of the states $\left\{\left|e_{0}, g_{0}, 0,0\right\rangle,\left|g_{L}, g_{0}, 0,1\right\rangle,\left|g_{L}, e_{R}, 0,0\right\rangle,\left|g_{R}, g_{0}, 1,0\right\rangle,\left|g_{R}, e_{L}, 0,0\right\rangle\right\}$ are almost zero during the whole dynamics. Figure 2(c): error probability $P_{e}(t)$ defined by Eq. (8). Figure 2(d): the probability $P_{p}$ with which one photon appears in the cavity. Figure $2(\mathrm{e})$ : the population $P_{e a}$ of the atoms in excited state $\left|e_{i}\right\rangle(i=L, 0, R)$.

\section{CONCLUSION}

In summary, based on the STIRAP and f-STIRAP techniques, we have proposed two schemes to generate entanglement of two ${ }^{87} R b$ atoms in a bi-mode cavity. According to the simulation results, in the schemes for creation of atomic entanglement states, the cavity mode, and the atomic excited states are nearly not populated, so these schemes are hardly influenced by the atomic spontaneous emission and the cavity decay. The error probability is also very small during the process.

Acknowledgments: This work was funded by Natural Science Foundation of China (Grant No 60677044).

[1] C. H. Bennett, G. Brassard, C. Crepeau, R. Jozsa, A. Peres, W. K. Wootters, Phys. Rev. Lett. 70,1895 (1993).

[2] C. H. Bennett, S. J. Wiesner, Phys. Rev. Lett. 69, 2881 (1992).

[3] H. de Riedmatten, I. Marcikic, H. Zbinden and N. Gisin, Quantum Information and Computation 2, 425 (2002).

[4] M. Zukowski, A. Zeilinger, M. A. Horne, Phys. Rev. A 55, 2564 (1997).

[5] G. Molina-Terriza, A. Vaziri, et.al. Phys. Rev. Lett. 92, 167903 (2004).

[6] T. C. Ralph, K. J. Resch, and A. Gilchrist, Phys. Rev. A 75, 022313 (2007).

[7] G Molina-Terriza, JP Torres, L Torner, Nature Physics 3, 305 (2007).

[8] A. Cabello, J. Mod. Opt. 5010049 (2003).

[9] F. Francica, S. Maniscalco, J. Piilo, F. Plastina, and K.-A. Suominen, Phys. Rev. A 79, 032310 (2009).

[10] A. Delgado, C. Saavedra, J.C. Retamal, Physics Letters A, 370, 22, (2007).

[11] X. B. Zou, K. Pahlke, and W. Mathis, Phys. Rev. A 67, 044301 (2003). 


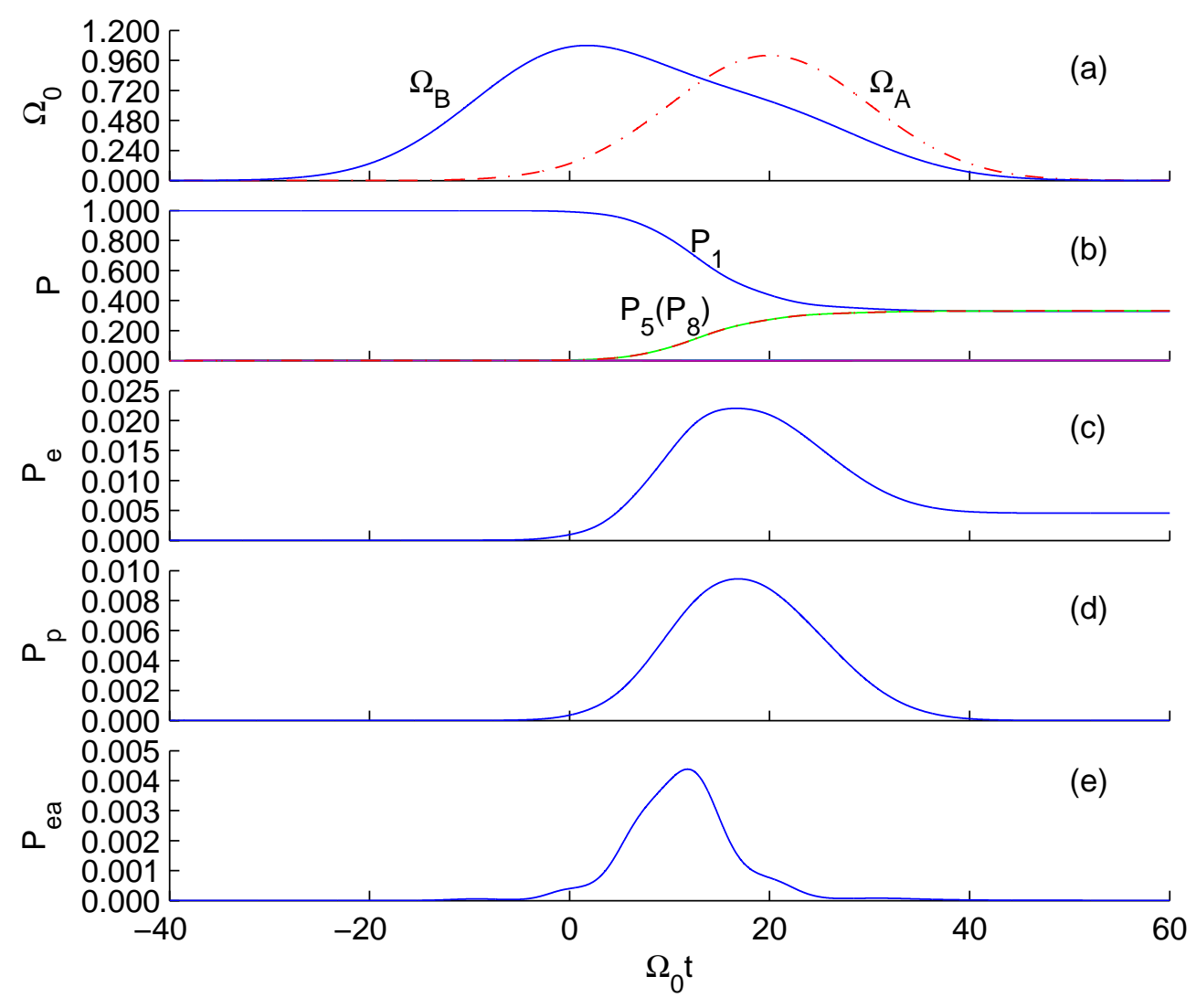

FIG. 3: The simulation results of the two-qutrit entanglement generation process, where we choose $g, \Omega_{0}, \tau, \kappa$, and $\gamma$ the same as in Figure 2. Figure 3(a): the Rabi frequency of $\Omega_{A}(t), \Omega_{B}(t)$. Figure 3(b): the time evolution of populations, where $P_{1}, P_{5}$, and $P_{8}$ mean the same as in Fig. 2. Figure 3(c): error probability $P_{e}(t)$ defined by Eq. (8). Figure 3(d): the probability $P_{p}$ with which one photon appears in the cavity. Figure $3(\mathrm{e})$ : the probability $P_{e a}$ of the atoms in excited state $\left|e_{i}\right\rangle(i=L, 0, R)$.

[12] S. B. Zheng, Phys. Rev. A 68, 035801 (2003).

[13] S. B. Zheng, Commun. Theor. Phys. 45, 539 (2006).

[14] S. B. Zheng, Chin. Phys. Lett. 22, 3064 (2005).

[15] X. M. Lin, Z. W. Zhou, Y. C. Wu, C. Z. Wang, and G. C. Guo, Chin. Phys. Lett. 22, 1318 (2005).

[16] G.W. Lin, M.Y. Ye, L.B. Chen, Q.H. Du and X.M. Lin, Phys. Rev. A 76 (2007).

[17] Asoka Biswas and G. S. Agarwal, J. Mod. Opt. 51, 1627 (2004).

[18] X. W. Wang and G. J. Yang, Phys. Rev. A 78, 024301 (2008).

[19] I. E. Linington and N. V. Vitanov, Phys. Rev. A 77, 010302 (2008).

[20] X.H. Huang, G.W. Lin, M.Y. Ye, Y.X. Tang and X.M. Lin, Opt. Commun. 281 (2008).

[21] Jian Li, K. Chalapat, and G. S. Paraoanu, Phys. Rev. B 78, 064503 (2008).

[22] A. Fay, E. Hoskinson, F. Lecocq, L. P. Léy, F. W. Hekking, W. Guichard, and O. Buisson, Phys. Rev. Lett. 100, 187003 (2008).

[23] D. Vitali, P. Tombesi, M. J. Woolley, A. C. Doherty, and G. J. Milburn, Phys. Rev. A 76, 042336 (2007).

[24] G. Chen, Z. Chen, L. Yu, and J. Liang, Phys. Rev. A 76, 024301 (2007).

[25] G. Vallone, E. Pomarico, F. D. Martini, P. Mataloni, and M. Barbieri, Phys. Rev. A 76, 012319 (2007).

[26] F. W. Sun, J. M. Cai, J. S. Xu, G. Chen, B. H. Liu, C. F. Li, Z. W. Zhou, and G. C. Guo, Phys. Rev. A 76,052303 (2007).

[27] G. E. Murgida, D. A. Wisniacki, and P. I. Tamborenea, Phys. Rev. B 79, 035326 (2009).

[28] S. K. Saikin, C. Emary, D. G. Steel, and L. J. Sham, Phys. Rev. B 78, 235314 (2008).

[29] Oreg, J., F. T. Hioe, and J. H. Eberly, Phys. Rev. A 29, 690 (1984).

[30] Gaubatz, U., P. Rudecki, M. Becker, S. Schiemann, M. Külz, and K. Bergmann, Chem. Phys. Lett. 149, 463 (1988).

[31] Gaubatz, U., P. Rudecki, S. Sciemann, and K. Bergmann, J. Chem. Phys. 92, 5363 (1990).

[32] P. Král, I. Thanopulos, and M. Shapiro, Rev. Mod. Phys. 79, 53 (2007).

[33] K Bergmann, H Theuer, and BW Shore, Rev. Mod. Phys. 70, 1003 (1998).

[34] Z. Kis and F. Renzoni, Phys. Rev. A 65, 032318 (2002).

[35] H. Goto and K. Ichimura, Phys. Rev. A 70, 012305 (2004).

[36] X. Lacour, N. Sangouard, S. Guérin, and H. R. Jauslin, Phys. Rev. A 73, 042321 (2006). 
[37] D. MØller, LB Madsen, and K. MØlmer, Phys. Rev. A 75, 062302 (2007).

[38] M. Hennrich, T. Legero, A. Kuhn, and G. Rempe, Phys. Rev. Lett. 85, 4872 (2000).

[39] S. Y. Ye, Z. R. Zhong, S. B. Zheng, Phys. Rev. A 77, 014303 (2008).

[40] S. Y. Ye, S. B. Zheng, Opt. Commun. 281, 1306 (2008).

[41] J. Song, Y. Xia and H. S. Song, J. Phys. B: At. Mol. Opt. Phys. 40, 4503 (2007).

[42] S. K. Saikin, C. Emary, D. G. Steel, L. J. Sham, Phys. Rev. B 78, 235314 (2008).

[43] L. Wang, X. L. Song, A. J. Li, H. H. Wang, X. G. Wei, Z. H. Kang, Y. Jiang, and J. Y. Gao, Opt. Lett. 33, 2380 (2008).

[44] N. V. Vitanov, K. A. Suominen, and B. W. Shore, J. Phys. B 32, 4535 (1999).

[45] M. Amniat-Talab, S. Guérin, N. Sangouard, and H. R. Jauslin, Phys. Rev. A 71, 023805 (2005); M. Amniat-Talab, S. Guérin, and H. R. Jauslin, Phys. Rev. A 72, 012339 (2005).

[46] J. Klein, F. Beil, and T. Halfmann, Phys. Rev. Lett. 99, 113003 (2007).

[47] L. B. Chen, M. Y. Ye, G. W. Lin, Q. H. Du, and X. M. Lin, Phys. Rev. A 76, 062304 (2007).

[48] H. H. Wang, L. Wang, X. G. Wei, Y. J. Li, D. M. Du, Z. H. Kang, Y. Jiang, and J. Y. Gao, Appl. Phys. Lett. 92, 041107 (2008).

[49] T Wilk, SC Webster, A Kuhn, G Rempe, Science 317, 488 (2007).

[50] B. Weber, H. P. Specht, T. Mueller, J. Bochmann, M. Muecke, D. L. Moehring, G. Rempe, Phys. Rev. Lett. 102, 030501 (2009).

[51] S. M. Spillane, T. J. Kippenberg, K. J. Vahala, K. W. Goh, E. Wilcut, and H. J. Kimble, Phys. Rev. A 71, 013817 (2005).

[52] K. M. Birnbaum, A. S. Parkins, and H. J. Kimble, Phys. Rev. A 74, 063802 (2006).

[53] H. Goto and K. Ichimura, Phys. Rev. A 70, 012305 (2004). 